\title{
Editorial
}

\section{Reviewers for 2021}

Ghana Med J 2020; 55(4): 238-239 doi: http://dx.doi.org/10.4314/gmj.v55i4.1

We are very grateful to all our reviewers for recommending manuscripts for publication. Our reviewers give of their time and expertise to the service of science. We recognise those who reviewed for us in 2021. We hope that many more will respond to our invitation in the coming years.

\begin{tabular}{|c|c|}
\hline Abantanga, Francis: Ghana & Bello, Ajediran: Ghana \\
\hline Ablordey, Anthony: Ghana & Beyuo, Vera: Ghana \\
\hline Abokyi, Samuel: Ghana & Biritwum, Richard: Ghana \\
\hline Adam, Ahmed: South Africa & Boahen, Eric: Ghana \\
\hline Adanikin, Abiodun: Nigeria & Boateng, Yaw: Ghana \\
\hline Addo, Edwina Beryl: Ghana & Boima, Vincent: Ghana \\
\hline Addo, Kennedy: Ghana & Bonsu, Frank: Ghana \\
\hline Adedia, David: Ghana & Boskabadi, Hassan: Iran \\
\hline Adeko, Oluseun: Nigeria & Cannegieter, Suzanne: Netherlands \\
\hline Adeniran, Abiodun: Nigeria & Chernyshov, P. V.: Ukraine \\
\hline Adjei, Patrick: Ghana & Chichom-Mefire, Alain: Cameroon \\
\hline Adokiya, Martin: Ghana & Clegg-Lamptey, Joe Nat: Ghana \\
\hline Adu-Aryee, Nii-Armah: Ghana & Dakubo, Jonathan: Ghana \\
\hline Afaa, Taiba: Ghana & Dalaba, Maxwell A.: Ghana \\
\hline Afriyie-Mensah, Jane: Ghana & Darko, Kwame: Ghana \\
\hline Agbley, Daniel: Ghana & Dedey, Florence: Ghana \\
\hline Agwu, Ezera: Uganda & Der, Edmund: Ghana \\
\hline Agyei-Nkansah, Adwoa: Ghana & Dogbe, Edith: Ghana \\
\hline Ahadzi, Dzifa: Ghana & Donkor, Peter: Ghana \\
\hline Aheto, Justice Moses K.: Ghana & Dordoye, Eugene: Ghana \\
\hline Aikins, Moses: Ghana & Duah, Amoako: Ghana \\
\hline Akaba, Godwin: Nigeria & Dzefi-Tettey, Klenam: Ghana \\
\hline Akakpo, Patrick : Ghana & Edwin, Frank: Ghana \\
\hline Akhiwu, Benjamin: Nigeria & Edzie, Emmanuel K. Mesi: Ghana \\
\hline Akpalu, Josephine: Ghana & Ekem, Ivy: Ghana \\
\hline Alagbe, Olayemi: Nigeria & Ekpe, Eyo: Nigeria \\
\hline Amissah-Arthur, Maame: Ghana & El-Gilany, Abdel-Hady: Egypt \\
\hline Amoakoh-Coleman, Mary: Ghana & Enimil, Anthony: Ghana \\
\hline Amoateng, Patrick: Ghana & Essuman, Akye: Ghana \\
\hline Amoyaw-Asamoah, Isabella: Ghana & Etwire, Victor: Ghana \\
\hline Anane-Fenin, Betty: Ghana & Fadare, Joseph: Nigeria \\
\hline Andoh-Adjei, Francis-Xavier: Ghana & Fasina, Oluyemi: Nigeria \\
\hline Ansong, Daniel: Ghana & Feldman, Steven: United States \\
\hline Antwi, Sampson: Ghana & Fiagbe, Delali: Ghana \\
\hline Anyanechi, Charles: Nigeria & Fiakpornoo, Martina: Ghana \\
\hline Aryeetey, Richmond: Ghana & Fofie, Chris: Ghana \\
\hline Asaleye, Christianah: Nigeria & Forson, Audrey: Ghana \\
\hline Asare Quansah, Gloria: Ghana & Gezawa, Ibrahim: Nigeria \\
\hline Asare, Offei: Ghana & Gumanga, Solomon: Ghana \\
\hline Asuquo, Marcus: Nigeria & Gupta, Amod: India \\
\hline Atta, Dzifa: Ghana & Gyedu, Adam: Ghana \\
\hline Awuku, Yaw: Ghana & Hesse, Afua: Ghana \\
\hline Ayettey-Adamafio, Mary: Ghana & Ho, Roger C.: Singapore \\
\hline Ayisi-Boateng, Nana: Ghana & Hodgson, Abraham: Ghana \\
\hline Baah, Winfred: Ghana & Idowu, Bukunmi: Nigeria \\
\hline Badoe, Ebenezer: Ghana & Igboeli, Nneka: Nigeria \\
\hline Baidoo, Kenneth: Ghana & Isara, Alphonsus: Nigeria \\
\hline Balcioglu, Yasin Hasan: Turkey & Issah, Kofi : Ghana \\
\hline
\end{tabular}

James, Peter: Australia Johnston, Carolyn: United States Kalu, Michael E.: Canada Kelishadi, Roya: Ghana Kenu, Ernest: Ghana Kerr, Peter G: Australia Khalifa, Ahmed: Egypt Kilba, Charlyne: Ghana Kitcher, Emmanuel: Ghana Klufio, George: Ghana Kokuro, Collins: Ghana Koram, Kwadwo: Ghana Kretchy, Irene: Ghana Kwakyi, Edward: Ghana Kyei, Mathew: Ghana Lamptey, Roberta: Ghana Langhammer, Birgitta: Norway Larsen-Reindorf, Rita: Ghana Lartey, Margaret: Ghana Lee, Yew Kong: Malaysia Lentoor, Antonio: South Africa Lim, Rosemary H.: United Kingdom Maged, Ahmed M.: Egypt Mahmoudabadi, Ali Zarei: Ghana Malm, Keziah: Ghana Mante, Sunny: Ghana Martinez, Edson Zangiacomi: Brazil Mate-Kole, Charles: Ghana Mektrirat, Raktham: Thailand Mensah, Samuel: Ghana Mensah, Yaw: Ghana Meysamie, Alipasha: Iran Michael, Godpower: Nigeria Mishra, Jiten: India Mohd Noor, Noor Haslina: Malaysia Morhe, Emmanuel: Ghana Morjaria, Priya: United Kingdom Motilewa, Olugbemi: Nigeria Mould-Millman, Nee-Kofi: United States Mume, Celestine: Nigeria Mustapha, Shettima : Nigeria Mutalub, Yahkub: Nigeria Mutambirwa, Shingai: South Africa Nartey, Edmund: Ghana Nasseri, Karim: Iran Ndlovu, Kwazi: South Africa Newman, Mercy: Ghana Nguah, Samuel: Ghana 


\section{Editorial}

Nkyekyer, Kobina: Ghana

Nsaful, Josephine: Ghana

Nsaful, Kwesi: Ghana

Nsiah, Kwabena: Ghana

Ntsiea, Mokgobadibe: South Africa

Nuertey, Benjamin: Ghana

Obadeji, Adetunji: Nigeria

Obadeji, Adetunji: Nigeria

Obiri-Yeboah, Dorcas: Ghana

Odeniyi, Ifedayo: Nigeria

Odugbemi, Babatunde: Nigeria

Ofori-Adjei, David: Ghana

Ogunbode, Adetola: Nigeria

Ogunnubi , Oluseun: Nigeria

Ohene, Sally-Ann: Ghana

Okonkwo, Ogugua: Nigeria

Okyere, Perditer: Ghana

Olagunju, Andrew: Nigeria

Olasode, Olayinka: Nigeria

Ologe, Foluwasayo: Nigeria

Olowookere, Samuel: Nigeria

Oppong, Samuel: Ghana

Ossai, Edmund Ndudi: Nigeria
Owusu, Enid: Ghana

Owusu, Michael: Ghana

Owusu-Agyei, Seth: Ghana

Page, Andrew: Australia

Palmieri, Patrick: Peru

Peltonen, Markku: Finland

Phillips, Richard: Ghana

Platonov, Pyotr: Sweden

Prazeres, Filipe: Portugal

Quarde, Akuffo: United States

Quashie, Neils B.: Ghana

Quayson, Solomon: Ghana

Reich, Adam: Poland

Renner, Lorna: Ghana

Sagoe-Moses, Isabella: Ghana

Saheeb, Birch: Nigeria

Samba, Ali: Ghana

Sarfo, Fred: Ghana

Seneadza, Nana: Ghana

Shaki, Omna: India

Shenoy, Suchitra: India

Shittu, Akeem: Nigeria

Souza, Dziedzom K. De: Ghana
Tachi, Kenneth: Ghana

Tagbor, Harry: Ghana

Takure, Augustine: Nigeria

Technau, Karl-Gunter: South Africa

Tettey, Mark: Ghana

Torpey, Kwame: Ghana

Tuoyire, Derek: Ghana

Udofia, Emilia: Ghana

Ulzen-Appiah, Kofi: Ghana

Umeora, O.U.J.: Nigeria

Umuerri, Ejiroghene: Nigeria

Wang, Leyi: United States

Whiting, Susan J.: Canada

Yahaya, James: Uganda

Yang, Hongmei: United States

Yawson, Alfred: Ghana

Yeboah-Manu, Dorothy: Ghana

Yorke, Ernest: Ghana

Zewde, Yared: Ethiopia

Editorial Office

Ghana Medical Journal

E-mail: editor@ghanamedj.org 